\title{
DEDDWTE

\section{8) Identificação de risco nutricional em idosos: malnutrition screening tool versus miniavaliação nutricional}

Vanessa Teles Felinto Mello'

A desnutrição é uma doença de grande prevalência no público idoso porém frequentemente não é diagnosticada, favorecendo ao maior risco de complicações hospitalares, além de maior tempo de internação e aumento dos gastos. A aplicação de métodos de rastreio nutricional torna-se importante para um diagnóstico precoce desse quadro, com condutas mais efetivas para seu tratamento. Objetivo: Verificar a capacidade de identificação de risco nutricional pelo Malnutrition Screening Tool em comparação à Miniavaliação nutricional em idosos e identificar a prevalência de risco nutricional e desnutrição no público avaliado. Métodos: Estudo transversal, analítico, que consistiu na realização de triagem e avaliação nutricional dos pacientes idosos ( $\geq 60$ anos) hospitalizados, através dos métodos Malnutrition Screening Tool e Miniavaliação nutricional. Resultados e Discussão: Foram avaliados 106 pacientes, com $48,1 \%$ apresentando risco nutricional e 22,6\% apresentando desnutrição segundo a Miniavaliação nutricional. Pelo Malnutrition Screening Tool, encontrou-se risco nutricional em 52,8\% dos pacientes nas primeiras 48 horas de internação. Em relação à capacidade do Malnutrition Screening Tool em identificar risco nutricional nos pacientes idosos, em comparação com a Miniavaliação nutricional, observa-se associação entre os testes $(\mathrm{p}<0,02)$, porém com tendência a um falso negativo, pois $19,8 \%$ dos pacientes classificados como sem risco nutricional pela Malnutrition Screening Tool apresentaram risco nutricional pela Miniavaliação nutricional. Conclusão: A utilização dos dois métodos se confirma para aqueles com risco nutricional, não podendo ser estendida para os sem risco nutricional pela divergência entre as análises, podendo ter o falso negativo nas avaliações da escala Malnutrition Screening Tool.

Nutricionista e Mestre em Saúde do Idoso, pela Fundação de Ensino e Pesquisa em Ciências da Saúde do Distrito Federal (Fepecs). Endereço para correspondência: SMHN Quadra 03, conjunto A, Bloco 1 Edifício Fepecs. 70.710907. Brasília, DF, Brasil. Email: vanessatfelinto@gmail.com 